\title{
La evolución de la estatura en Francia y en España, 1770-2000. Balance historiográfico y nuevas evidencias
}

\author{
José Miguel Martínez-Carrión (*) y Javier Puche-Gil (*) \\ (*) Departamento de Economía Aplicada, Universidad de Murcia. jcarrion@um.es
}

Dynamis

[0211-9536] 2011; 31 (2): 429-452
Fecha de recepción: 13 de marzo de 2010

Fecha de aceptación: 5 de mayo de 2010

SUMARIO: 1.-Introducción 2.-EI desarrollo de la antropometría en Francia 3.-La réplica española. 4.-La eclosión de la auxología y su influencia en la historiografía. 5.- La evolución de la estatura de los varones en Francia y en España. Una perspectiva comparada. 6.-Conclusiones.

RESUMEN: La estatura es utilizada por los pediatras y los antropólogos como medida de salud y bienestar de las poblaciones humanas. En las últimas décadas los historiadores la usan como indicador biológico del nivel vida para explorar el impacto producido por las transformaciones ambientales, sociales y económicas en el bienestar del pasado. Este artículo muestra las contribuciones históricas realizadas al tema por diferentes especialistas y compara la evolución de la estatura de los hombres en Francia y en España. Con datos de los reemplazos militares desde la década de 1770 hasta finales del siglo XX muestra los cambios seculares en ambos países y la existencia de ciclos como consecuencia de periodos de deterioro del estado nutricional.

PALABRAS CLAVE: Estatura, Francia, España, bienestar biológico.

KEY WORDS: Height, France, Spain, biological well-being.

\section{Introducción $(*)$}

La estatura humana ha sido objeto de estudio durante mucho tiempo por médicos pediatras y antropólogos, principalmente. El uso de los indicadores antropométricos cuenta con una larga tradición entre los especialistas de la salud pública y la antropología física que se remonta al siglo XIX.

(*) Este trabajo ha sido realizado en el marco de los Proyectos HAR2010-20684-C02-02, SEJ200767613 y BEC2002-03927 financiados por el MEC y el Ministerio de Ciencia y Tecnología (Gobierno de España). Los autores agradecen los comentarios de los evaluadores anónimos. 
Considerada como un indicador acumulativo del estado nutricional neto al final del crecimiento, la talla media de las poblaciones constituye una excelente medida del bienestar, la salud y el nivel de vida biológico. De acuerdo con James M. Tanner (1920-2010), uno de los especialistas más renombrados, «la talla es el espejo del nivel de vida de una sociedad» ${ }^{1}$. En las últimas décadas, también los historiadores emplean los indicadores antropométricos, sobre todo la estatura, para estudiar los cambios producidos en el tamaño de los cuerpos humanos, los factores ambientales y socioeconómicos determinantes y las relaciones que se establecen entre el crecimiento económico y el desarrollo humano desde tiempos del Neolítico. El avance del nuevo campo de estudio, que viene denominándose como «historia antropométrica», ha sido extraordinario ${ }^{2}$. Sus mayores logros, sin embargo, han sido desbrozar las consecuencias fisiológicas de la Revolución Industrial y el impacto ambiental ocasionado por las transformaciones sociales y económicas de los tres últimos siglos ${ }^{3}$.

El objetivo de este trabajo es doble, señalar la importancia de los tempranos estudios de antropometría en Francia y España y mostrar la evolución comparada de la estatura de los hombres adultos en dichos países. Con datos de los mozos llamados a filas provenientes de los quintos españoles y del reclutamiento militar francés, se construyen series históricas que permiten diagnosticar la tendencia secular y la existencia de ciclos de la estatura desde las cohortes de finales del siglo XVIII hasta finales del siglo XX. Los resultados sugieren que la estatura humana estuvo sujeta a las circunstan-

1. Tanner, James. Introduction: Growth in height as a mirror of the standard of living. In: Komlos, John, ed. Stature, living standards, and economic development. Essays on Anthropometric history. Chicago: The University of Chicago Press; 1994, p. 1-9. Similar enfoque puede encontrarse en Martorell, Reynaldo; Habicht, Jean-Pierre. Growth in early childhood in developing countries. In: Falkner, Tanner, eds. Human growth: A comprehensive treatise. New York: Plenum; 1986, vol. 3, p. 241-262.

2. Komlos, John; Baten, Jorg, eds. Special Issue: Recent research in Anthropometric History. Social Science History. 2004; 28 (2): 191-350. Steckel, Richard. Heights and human welfare: Recent developments and new directions. Explorations in Economic History. 2009; 46: 1-23.

3. Fogel, Robert. The escape from hunger and premature death, 1700-2100. Europe, America and the Third World. Cambridge: Cambridge University Press; 2004. Floud, Roderick; Watcher, Kenneth; Gregory, Annabel. Height, health and history: Nutritional status in Britain, 1750-1980. Cambridge: Cambridge University Press; 1990. Komlos, John, ed. The biological standard of living on three continental. Further explorations in anthropometric history. Oxford: Westview Press; 1995. Steckel, Richard H; Rose, Jerome C, eds. The backbone of history. Health and nutrition in the Western hemisfere. Cambridge: Cambridge University Press; 2002. 
cias ambientales y a las transformaciones políticas y socioeconómicas que registraron los dos países en la etapa contemporánea.

\section{El desarrollo de la antropometría en Francia}

El historiador francés Emmanuel Le Roy Ladurie fue el primero que usó la estatura para indagar aspectos sociales y económicos de las poblaciones históricas. En 1969, en pleno auge de la escuela francesa de Annales, Le Roy Ladurie y su equipo mostraron fuertes contrastes de los promedios de estatura según la educación y la circunscripción departamental de los reclutas en Francia durante la primera mitad del siglo XIX. Los soldados franceses que sabían leer y escribir en la década de 1840 eran 1,4 cm más altos que los soldados analfabetos ${ }^{4}$. Los resultados departamentales revelaron, luego, diferencias entre el norte y el sur de Francia y mostraron una correlación positiva entre talla y educación para las generaciones de 1820$30^{5}$. Las causas de estos contrastes se explicaron por las desigualdades en el acceso a la educación, dado que en Francia no era universal y sólo las familias con rentas más elevadas o que disfrutaban de cierto status social podían atender a la educación «primaria» de sus hijos. Aquellos estudios supusieron el nacimiento de la «historia antropométrica».

Pero la validez de la estatura y de los registros antropométricos en la medición de la salud y el bienestar de los niños y de los adultos en general había sido reconocida muy tempranamente en Francia. Los especialistas sitúan los orígenes de la auxología y de la antropología física en el país galo entre 1780 y $1830^{6}$. Las primeras pesquisas se remontan a las descripciones de Volney (1757-1820) aunque los primeros trabajos antropométricos realizados sobre la estatura se deben al conde Guéneau de Montbeillard (1720-1785) en 1759, a Jacques Tenon (1724-1816) en 1783 y a AntoineAudet d'Hargenvilliers (1768-1835) que, siendo jefe de negociado del reclutamiento francés, llamó la atención sobre la importancia de la estatura

\footnotetext{
4. Le Roy Ladurie, Emmanuel; Bernagean, Nicole; Pasquet, Yves. Le Conscrit et l'ordinateur: Perspectives de recherches sur les Archives Militaires du XIXe siècle francais. Studi Storichi. 1969; 10: 260-308.

5. Le Roy Ladurie, Emmanuel; Demonet, Michel. Alphabétisation et stature: Un tableau comparé. Annales: Économies, Sociétés, Civilisations. 1980; 35: 1329-32.

6. Ballester Añón, Rosa; Perdiguero Gil, Enrique. Ciencia e ideología en los estudios sobre crecimiento humano en Francia y en España (1900-1950). Dynamis. 2003; 23; 61-84.
} 
en los reclutamientos de la armada francesa en 1817. Pero los primeros «teóricos del desarrollo» fueron el barón Charles Dupin, cuya principal aportación se halla fundamentada entre 1826 y 1828, y Adolphe d'Angeville (1796-1856). Ambos fueron coetáneos del célebre médico higienista LouisRené Villermé (1782-1863) y, junto a otros científicos, dieron origen a dos tipos de interpretaciones, racial y social, que convivieron a mediados del siglo XIX ${ }^{7}$.

Considerado como el primer tratado de antropometría, la «Mémorie sur la taille de l'homme en France» publicada por Villermé, en Annales d'hygiène publique et de médecine légale en 1829, constituye un verdadero manifiesto del emergente movimiento higienista francés, que en buena parte vinculó los factores del crecimiento y de la desigualdad de las tallas a las condiciones sociales y los niveles de vida de las poblaciones. Junto al estudio de Villermé, destacó el de Adolphe Quetelet (1796-1874) sobre la estatura de los niños en Bélgica ${ }^{8}$. El conjunto de estas primeras investigaciones antropométricas mostró la variabilidad y la diversidad biológica de las poblaciones humanas, por grupos sociales y por circunscripciones territoriales.

En el tercer cuarto del siglo XIX el desarrollo de la antropometría se fortaleció por la consolidación de la antropología nacional francesa. Aunque las bases sociales y económicas inspiraron el trabajo de Villermé y de otros científicos franceses, la interpretación racial se impuso en los medios intelectuales franceses por los historiadores liberales de comienzos del siglo XIX que contribuyeron a vulgarizar la idea de raza. El auge de la tesis racial se debió a la posición privilegiada que Paul Broca (1824-1880), Jean Christian Marc Boudin (1806-1867) y Jacques Bertillon (1821-1883), entre otros, ocuparon en los medios académicos. El primero es el precursor de la escuela francesa de antropología física: creó el Laboratoire d'Anthropologie de

7. Sobre los comienzos de la antropometría en Francia y las escuelas que se desarrollaron posteriormente, ver Heyberger, Laurent. La révolution des corps. Décroissance et croissance staturale des habitants des villes et des campagnes en France, 1780-1940. Strasbourg y Belfort: Presses universitaires de Strasbourg \& Université de technologie Belfort-Montbéliard; 2005

8. Arquiola, Elvira. Anatomía y antropología en el positivismo francés. Asclepio. 1979; 30-31: 19-32. Sobre la importancia de su contribución a la epidemiología social: Chantal, Julia; Valleron, Alain-Jacques. Louis-René Villermé (1782-1863), a pioneer in social epidemiology: Re-analysis of his data on comparative mortality in Paris in the early 19th century. Journal of Epidemiology Community Health. 2010; 64: 1. 
l'Ecole des Hautes Etudes en 1868, la Revue d'Anthropologie en 1872 y la Ecole d'Anthropologie de París en 1876. Como fundador de la Société d'Anthropologie de Paris, editó asimismo dos publicaciones de enorme relevancia posterior: las Mémories de la Société d'Anthropologie de Paris (1859) y los Bulletins de la Société d'Anthropologie de Paris (1859). Entre los especialistas de entonces predominaban las teorías raciales que explicaban el comportamiento y la variabilidad de las estaturas. La genética pesaba más que los factores ambientales que sólo comenzaron a barajarse tibiamente a finales del siglo XIX. ${ }^{9}$ La aparición de la especialidad pediátrica en estas décadas también alentó la medición antropométrica, al plantear una mayor preocupación por los temas del crecimiento y que el niño fuera objeto privilegiado del conocimiento científico. Como ha señalado Ballester, obras de consulta como el Dictionnaire Encyclopédique des Sciences Médicales (desde 1879) recogen voces propias: accroissement, croissance y taille, exponente claro de la importancia concedida al tema, y se difunde el mécomètre, instrumental de medición de la talla, tambien de creación francesa ${ }^{10}$.

Desde comienzos del siglo XX las tesis sociales y económicas que explicaban el comportamiento de la estatura fueron ganando adeptos frente a las tesis raciales que comenzaron a ser cada vez más discutidas, alcanzando aquéllas su plenitud a mediados del siglo XX. Los primeros trabajos de teoría social se debieron al periodista y librepensador Jules Carret (18441912) y prosiguieron con la labor de los pediatras cada vez más presentes en los debates sobre el crecimiento humano y en particular sobre el de los niños y de los adolescentes ${ }^{11}$. Mediaron entre tanto planteamientos como la influencia del ferrocarril en el crecimiento de la estatura, que entusiasmó a algunos científicos entre los años 1880 y 1890, y las mejoras de la alimentación y de las condiciones de vida que llevaron casi parejas el empuje industrial y el desarrollo económico. La controversia entre las tesis ambientales y las raciales cobró fuerza en el periodo de entreguerras $\mathrm{y}$, aunque las primeras se fueron imponiendo como tesis explicativas del crecimiento de las estaturas, las tesis raciales, básicamente hereditarias,

9. Heyberger, n. 7, p. 48-77.

10. Sobre el papel de la medicina y de la pediatría en la mejora del conocimiento de las enfermedades y el crecimiento infantiles en este periodo, ver Ballester Añón, Rosa. La historia clínica pediátrica durante el siglo XIX. Zaragoza: Universidad; 1977, y Seidler, Eduard. El desarrollo de la pediatría moderna. In: Laín Entralgo, Pedro. Historia universal de la medicina. Barcelona: Salvat; 1981, vol. 6, p. 203-215.

11. Ballester; Perdiguero, n. 6, p. 75 y ss. 
mantuvieron alguna influencia hasta comienzos de la década de 1960. El último y mejor exponente de las últimas fue Henri Vallois (1889-1981), antropólogo, paleontólogo y médico, que pudo difundirlas desde su influyente puesto como director del Laboratorio de Antropología del Musée de l'Homme $(1938)^{12}$. Paradójicamente, desde 1964 diversas publicaciones auspiciadas por el mismo centro, el laboratorio de Vallois, y publicadas por la Société d'Anthropologie de Paris otorgaron protagonismo a los factores económicos y sociales. De ese modo, la antropometría, la auxología y la antropología física francesas abrieron nuevas perspectivas y enfoques que revalidaban el rol de las tesis sociales o de los factores ambientales en el crecimiento humano.

\section{La réplica española}

La réplica española a los tempranos estudios de antropometría se encuentra entre los médicos higienistas. Gregorio Andrés Espala ${ }^{13}$, Francisco Bona ${ }^{14}$, Anastasio Chinchilla ${ }^{15}$ y otros responsables de la sanidad militar española, utilizaron datos de estaturas y valoraron la importancia de dotarse de estadísticas, que en España se publicaron regularmente desde $1858^{16}$. A finales del siglo XIX, la antropología física española se desarrolló gracias a los estudios de Telesforo Aranzadi (1860-1945) y Luis de Hoyos-Sainz $(1868-1951)^{17}$. Por entonces, la Real Academia de Ciencias Políticas y Mo-

12. Vallois, Henri. Anthropologie de la population française. Toulouse: Didier; 1943.

13. Andrés y Espala, Gregorio. Reflexiones sobre la talla, peso y perímetro torácico. La Gaceta de Sanidad Militar. 1877; 3: 1-7.

14. Bona, Francisco. Estadística física de las tallas y de los defectos físicos. Revista General de Estadística. 1863; 2: 305-321.

15. Chinchilla, Anastasio. Estadística de Sanidad Militar. Revista General de Estadística. 1864; 3: 1-31.

16. En las publicaciones militares españolas de la época se tradujeron estudios realizados por militares europeos que manifestaban el deterioro fisiológico de los reclutamientos entre 1850 y 1870. Tal vez estas proclamas tenían como objetivo crear un clima favorable para reducir la edad de reclutamiento y rebajar las tallas mínimas que regulaban el acceso a los ejércitos nacionales, ver Tschouriloff, Michel. Estudio sobre la degeneración fisiológica de los pueblos civilizados (causas de la degeneración). La Gaceta de Sanidad Militar. 1877; 3: 45-52, 109-115, 192-199, 276-283, 301-305, 363-366 y 384-392.

17. Sobre la contribución de estos autores, puede consultarse la obra de Ortiz García, Carmen. Luis de Hoyos Sainz y la Antropología Española. Madrid: CSIC; 1987. Ortiz García, Carmen; Sánchez Gómez, Luis Ángel, eds. Diccionario histórico de la antropología española. Madrid: CSIC; 1994, p. 100-103 y 525-527. 
rales acogió sendos discursos del economista y reputado político Laureano Figueroa (1816-1903) y del médico y antropólogo Federico Olóriz Aguilera (1855-1912) que versan sobre la estatura de los españoles ${ }^{18}$. Por su parte, Pío Suárez Inclán y Luis Sánchez Fernández abordaron los problemas del reclutamiento español y las características antropométricas de los españoles en el cambio de siglo ${ }^{19}$.

Con el avance de la pediatría española en las primeras décadas del siglo XX, los especialistas pudieron realizar estudios sobre el crecimiento infantil con herramientas auxológicas. Durante el siglo XIX se sentaron las bases metodológicas y conceptuales para abordar el crecimiento infantil, pero fue a partir de las primeras décadas del siglo XX cuando se desarrolló la pediatría y creció el número de publicaciones en Europa. Muchas de las publicaciones europeas incluyeron comparaciones de tallas y pesos en niños de las mismas edades y hasta de los diferentes países, lo que permitió ampliar el conocimiento de las distintas fases del crecimiento infantil en distintos contextos ambientales. Sin embargo, en España la mayor parte de los trabajos científicos que incluían mediciones de tallas y otras medidas antropométricas provenían de médicos que tenían una clara vertiente higiénico-sanitaria o de aplicación clínica de comienzos del siglo XX. La institucionalización de las medidas antropométricas se realizó dentro de los esquemas de la higiene escolar, tarea en la que destacan los trabajos de Martínez Vargas y Súñer ${ }^{20}$. Los debates se centraron mayormente en erradicar la mortalidad infantil y estuvieron más cercanos al discurso de los movimientos de medicina social y eugenesia, propios de las luchas sanitarias de principios de siglo XX y de los movimientos de protección a la infancia ${ }^{21}$.

18. Figuerola, Laureano. La talla de los mozos para el servicio militar sorteados y medidos en las quintas de 1858 a 1867. Memorias de la Real Academia de Ciencias Políticas y Morales. 1893; 7: 305-311. Olóriz Aguilera, Federico. La talla humana en España. Discurso leído en la Real Academia de Medicina. Madrid: Imprenta Nicolás Moya; 1896, p. 4-61.

19. Martínez-Carrión, José Miguel; Pérez-Castejón, Juan José. On the height of Spanish recruits during the early phases of modern economic growth. Jarbuch fur Wirtschaftgeschichte. 2000; 1: 159-176.

20. Ballester Añón, Rosa; Perdiguero Gil, Enrique. Los estudios sobre crecimiento humano como instrumento de medida de la salud de los niños españoles. Áreas. Revista de Ciencias Sociales, 2000; 20: 161-170. Ballester Añón, Rosa. Deformaciones corporales en la pediatría española. Tradición y ciencia ante el desarrollo de la infancia, 1800-1930. Cuadernos de Historia Contemporánea. 2002; 24: 189-207.

21. Ballester Añón, Rosa; Balaguer, Emilio. La infancia como valor y como problema en las luchas sanitarias de principios de siglo en España. Dynamis. 1995; 15: 177-192. Rodríguez Ocaña, 
La institucionalización de las medidas antropométricas en España se produjo durante la primera mitad del siglo $\mathrm{XX}^{22}$. Los efectos ocasionados en la nutrición infantil por la Guerra Civil de 1936-39 y la larga posguerra promovieron el interés de los especialistas por las mediciones antropométricas y la elaboración de curvas de crecimiento en niños de diferentes rangos. Con el apoyo de la Fundación Rockefeller, Grande Covián, Jiménez García, Rof Carballo, entre otros, realizaron numerosos estudios clínicos sobre los estados de desnutrición observados durante la guerra y la posguerra y sobre la alimentación y el desarrollo físico de los niños ${ }^{23}$. Comprobaron una amplia difusión de la pelagra muy constante en los estados de desnutrición de las poblaciones madrileñas y observaron grandes deficiencias de vitaminas y carencias nutricionales en las ingestas de la guerra, que produjeron «debilidad muscular». Los estudios se completaron con patrones de desarrollo físico y se establecieron curvas y tablas de crecimiento por edades para determinar la variabilidad normal y analizar las condiciones alimenticias de distintos grupos socioeconómicos.

En plena autarquía, a mediados de la década de 1940, se publican diversos trabajos provenientes del campo de la pediatría que revelan el empleo de técnicas más sofisticadas de análisis de los datos antropométricos, entre los cuales destacan los de Sáinz de los Terreros, Mezquita y López, Royo y Vilar Caso. En general, mostraban resultados desalentadores al comparar las medidas antropométricas con las de otros países europeos. Los niños españoles eran notoriamente inferiores a la talla de los niños alemanes, franceses, belgas o británicos. De acuerdo con las teorías ya predominantes, para dichos autores las desigualdades sociales y económicas constituían un factor determinante, por encima incluso de los factores genéticos y étnicos ${ }^{24}$.

Esteban. La construcción de la salud infantil. Ciencia, medicina y educación en la transición sanitaria en España. Historia Contemporánea. 1999; 18: 19-52.

22. Ballester; Perdiguero, n. 20, p. 161 y ss.

23. Grande Covián, Francisco. Deficiencias vitamínicas en Madrid durante la Guerra Civil. Una reminiscencia. In: Estellés, José. Los médicos y la medicina en la Guerra Civil española. Madrid: Sanidad y Ediciones; 1986, p. 61-68. Jiménez Díaz, Carlos; Roda, Emilio; Castro Mendoza, H. Estudios sobre los estados de desnutrición. Medicina Clínica Española. 1942; 7 (1): 25-43. Jiménez García, Francisco. Estudio clínico de los trastornos carenciales observados en la población civil de Madrid durante la guerra (1936-1939). Revista Clínica Española. 1940; 1 (3): 231-237. Robinson, William; Janney, John H; Grande-Covián, Francisco. An evaluation of the nutritional status of a population group in Madrid, Spain, during the summer of 1941. Journal of Nutrition. 1942; 24: 557-584.

24. Ballester; Perdiguero, n. 20, p. 167. 


\section{La eclosión de la auxología y su influencia en la historiografía}

El auge de la auxología y la difusión de las mediciones antropométricas tuvieron lugar en el curso de la segunda mitad del siglo XX. El fenómeno, protagonizado por los antropólogos físicos y los pediatras principalmente, es observable tanto en Francia como en España. Entre las décadas de 1950 y 1970, los especialistas se volcaron en la medición del crecimiento humano - en plena fase de crecimiento económico y del mayor avance del bienestar en el mundo desarrollado- y enfatizaron la importancia de los factores ambientales. Frente a las tesis tradicionales que valoraban la herencia genética, las investigaciones mostraron el peso determinante de las variables socioeconómicas. De nuevo, adquirió protagonismo la escuela francesa de antropología física con los estudios de Marie-Claude Chamla y de sus colaboradores. Entre 1964 y 1977 se publicaron una serie de trabajos que abordaron el crecimiento de la talla, comparado con el de otros países de Europa, y las diferencias por clases sociales, status económico, profesional y residencia ${ }^{25}$. En España, durante las décadas de 1950 y 1960, se publicaron los primeros estudios que revelan el crecimiento de los españoles en el curso de la primera mitad del siglo $\mathrm{XX}^{26}$. Provenientes del campo de la antropología física se llevaron a cabo, a mediados de la década de 1970, análisis sobre la tendencia secular (secular trend) realizados por Cristina Bernis, aunque el Instituto Bernardino de Sahagún de Antropología y Etnología (Madrid) venía desarrollando desde la década de 1940 una intensa labor de investigación sobre el estado nutricional y la estatura de los muchachos españoles bajo la dirección de M. A. Fernández Cabeza $^{27}$. Desde el campo de la pediatría, el cambio de rumbo comienza

25. Chamla, Marie-Claude. L'accroissement de la stature en France de 1880 a 1960: comparaison avec les pays d'Europe occidentale. Bulletins et Mémoires de la Société d'Anthropologie de Paris. 1964; 6 (11): 201-278; Chamla, Marie-Claude; Marquer, Paulette. Stature et niveau économique en France. L'Anthropologie. 1961; 65: 277-280. Posteriormente, en Olivier, Guy. Biologie humaine et régions françaises. Comptes-Rendus de l'Académie des Sciences de Paris. 1969; 268: 2085-2087.

26. Muro, A; Aceña, A; Vivanco, F. Patrones de crecimiento de niños normales españoles. Revista Clínica Española. 1954; 53: 360-366. Palacios, J. M; Vivanco, F. Datos de talla y peso de 128.000 niños españoles. Revista Clínica Española. 1965; 99: 230-238. Palacios, J. M; García, A; Vivanco, F. El crecimiento de los niños españoles desde el nacimiento hasta los cinco años. Revista Clínica Española. 1970; 118: 419-424.

27. Bernis, Cristina. Sobre el aumento secular de la estatura en España. Trabajos de Antropología. 1976; 18: 27-32. Una revisión desde la antropología biológica en España en: Rebato, Esther. 
con la introducción de simples técnicas que posibilitan, en la década de 1950, el entendimiento de los procesos de crecimiento y la mejora de su medición por padres y maestros de escuela ${ }^{28}$.

En la década de 1970 la influencia de los trabajos de James M. Tanner y de los pediatras o especialistas en biología humana resultó decisiva ${ }^{29}$. En los años siguientes, una pléyade de antropólogos físicos y epidemiólogos mostró que las tallas humanas están influenciadas por la calidad de la nutrición y el impacto de las enfermedades ambientales. Los factores ecológicos, socioeconómicos y culturales se muestran tan decisivos o más que los factores genéticos. De acuerdo con ellos, las tallas más bajas expresan situaciones carenciales y malnutrición y revelan problemas ambientales, probablemente condicionados por la desigualdad social en el acceso a los recursos básicos ${ }^{30}$. Una alimentación insuficiente y la prevalencia de enfermedades durante la infancia pueden ralentizar el crecimiento de los niños y limitar el desarrollo mental en determinados casos. Análisis recientes revelan que la talla está asociada con una mejor salud física y mental y una mayor habilidad cognitiva al final de la vida ${ }^{31}$. Gozar de buena salud en la infancia podría correlacionar positivamente con los logros educacionales y el empleo en edades adultas. Por ello, el estatus social, la ocupación, la escolarización y los efectos de la educación de los padres en la nutrición, la desigualdad y el bienestar de los hijos son cuestiones atendidas por la nueva historia antropométrica.

Los avances de la auxología y la antropología biológica hicieron mella entre los historiadores económicos. Las pesquisas antropométricas de Le Roy Ladurie y sus discípulos fueron emuladas por diversos equipos de investigación formados por historiadores económicos que exploraron, desde comienzos de la década de 1980, las relaciones entre los niveles de vida biológicos y el desarrollo económico. Los hallazgos del historiador francés

The studies on secular trend in Spain: a review. In: Bodzsár, Éva B; Susanne, Charles, eds. Secular growth changes in Europe. Budapest: Eötvös University Press; 1998, p. 297-315.

28. Ballester; Perdiguero, n. 20.

29. Eveleth, Phyllis B.; Tanner, James M. Worldwide variation in human growth, Cambridge: Cambridge University Press; 1976; Tanner, James M. A history of the study of human growth. Cambridge: Cambridge University Press; 1981.

30. Bodzsár, Éva B; Susanne, Charles, eds. Secular growth changes in Europe. Budapest: Eötvös University Press; 1998.

31. Case, Anne; Paxson, Christina. Stature and status: Height, ability and labor market outcomes. Journal of Political Economy. 2008; 116 (3): 499-532. 
influyeron en el equipo de Robert Fogel (Universidad de Chicago), cuyo proyecto de investigación sobre las estaturas y el descenso de la mortalidad en el mundo occidental durante los tres últimos siglos fue patrocinado por el National Bureau of Economic Research (NBER) y el Center for Population Economics $^{32}$. En 1993 recibía el premio Nobel de Economía como reconocimiento a los trabajos cliométricos. En España, los primeros trabajos de historia antropométrica son de fechas relativamente tempranas. Así, en el III Congreso de la Asociación Española de Historia Económica, celebrado en Segovia en 1985, hubo una sesión dedicada a medidas antropométricas y niveles de vida. Desde entonces los avances han sido extraordinarios ${ }^{33}$.

Entre los principales temas abordados por la historia antropométrica destaca la evolución de las estaturas durante la revolución industrial. La cuestión ha suscitado una interesante controversia en Estados Unidos, similar a que tuvo lugar hace medio siglo con el nivel de vida en la industrialización inglesa que enfrentó a pesimistas y optimistas. Sin embargo, a diferencia de entonces, en la actualidad existe mucho más consenso, habida cuenta que todos los resultados publicados con evidencia antropométrica revelan el pesimismo en torno al bienestar biológico a comienzos de la industrialización. La literatura sobre el tema plantea que el bienestar biológico evolucionó inversamente al bienestar económico en los inicios del crecimiento económico moderno: la estatura disminuyó mientras incrementó la renta per capita ${ }^{34}$. En la mayor parte de los países, el deterioro de la estatura observado en las décadas centrales y en otros periodos del siglo XIX coincidió con crisis de subsistencias, guerras y episodios epidémicos, junto a importantes desplazamientos humanos, lo que probablemente extendió el alcance del fenómeno a áreas no industrializadas ${ }^{35}$.

32. Los primeros resultados aparecieron en Fogel, Robert W.; Engerman, Stanley L.; Floud, Roderick et al. Secular changes in American and British stature and nutrition. Journal of Interdisciplinary History. 1983; 14 (2): 445-481.

33. Ver un estado de la cuestión en Martínez-Carrión, José Miguel. La Historia Antropométrica y la historiografía Iberoamericana. Historia Agraria. 2009; 47: 11-18. Sobre Francia, ver el número monográfico: Heyberger, Laurent, dir. L'Historie anthropométrique en France. Histoire, Economie \& Société. 2009; 28 (1): 3-124.

34. Komlos, John. Shrinking in a growing economy? The mystery of physical stature during the industrial revolution. Journal of Economic History. 1998; 58 (3): 779-802. Steckel, Richard H.; Floud, Roderick. C., eds. Health and welfare during Industrialization. Chicago: Chicago University Press; 1997.

35. Ewert, Ulf Christian. The biological standard of living on the decline: Episodes from Germany during early industrialization. European Review of Economic History. 2006; 10: 51-88. 
La evolución de las tallas en el siglo XVIII y durante la crisis del Antiguo Régimen ha recabado el interés en los últimos años. La mayoría de las investigaciones muestran caídas más o menos importantes del estado nutricional entre 1770 y 1820 , consecuencia sobre todo de la presión malthusiana. El fuerte crecimiento demográfico y el aumento de los precios de los alimentos en la segunda mitad del siglo XVIII ocasionó el debilitamiento de las economías familiares y, junto a las violentas crisis de subsistencias que se sucedieron a finales de la centuria, desataron las hambrunas y las crisis de mortalidad en buen aparte de Europa ${ }^{36}$. Este último factor, el aumento de los precios de los alimentos, también condicionó el estado nutricional de las poblaciones americanas al final del periodo colonial ${ }^{37}$.

El impacto de las enfermedades, como el paludismo, el sarampión o la viruela ${ }^{38}$, y la influencia de los contextos ambientales en el mundo del trabajo, como la minería del carbón y del plomo ${ }^{39}$, han ocasionado importantes discusiones. La desigualdad y los procesos de convergencia de las tallas ${ }^{40}$, los determinantes de la estatura y otros indicadores del nivel de vida, como los salarios ${ }^{41}$, han impulsado numerosas investigaciones, que enlazan así con las primeras indagaciones acerca del impacto que el crecimiento económico y la industrialización ejercieron sobre la salud y la estatura. La talla de los nativos americanos, la esclavitud y la huella del colonialismo, la del trabajo infantil, los cambios institucionales, el impacto

36. A'Hearn, Brian A. Anthropometric evidence on living standards in Northen Italy, 1730-1860. Journal of Economic History. 2003; 63 (2), 351-381; Cinnerella, Franceso. Optimist or pessimists? A Reconsideration of nutritional status in Britain, 1740-1865. European Review of Economic History. 2008; 12 (3): 325-354; Komlos, John; Hau, Michael y Bourguinat, Nidas. An anthropometric history of early-modern France. European Review of Economic History 2003; 7: 153-189.

37. Challú, Amilcar. Agricultural crisis and biological well-being in Mexico, 1730-1835. Historia Agraria. 2009; 47: 21-44. Salvatore, Ricardo D.; Coastsworth, John H.; Challú, Amilcar E. (eds.). Living Standards in Latin Amercian history. Height, welfare, and development, 1750-2000. Cambridge, Harvard University Press; 2010.

38. Razzell, Peter. Did smallpox reduce height? Economic History Review. 1998; 51 (2): 351-359.

39. Kirby, Peter. Causes of short stature among coal-mining children, 1823-1850. Economic History Review. 1995; 48: 687-699.

40. Meisel, Adolfo; Vega, Margarita. The biological standard of living (and its convergence) in Colombia, 1870-2003. A tropical success story. Economics and Human Biology. 2007; 5: 100122. Komlos, John. Anthropometric evidence on economic growth, biological well-being and regional convergence in the Habsburg Monarchy, c. 1850-1910. Cliométrica. 2007; 1: 211-237.

41. Baten, Joerg. Heights and real wages in the 18th and 19th centuries: and international overview. Jarbuch für Wirtschaftgeschichte. 2000; 1: 29-42. 
de los regímenes autárquicos y autoritarios, el coste de las transiciones a nuevos regímenes o las desigualdades generadas desde entonces hasta los efectos de la globalización también han llamado la atención de los historiadores. La cuestión del género, insuficientemente tratada por el sesgo de las fuentes que siguen siendo principalmente militares, comienza a desarrollarse con nuevas bases de datos de estaturas de niñas y mujeres, que permiten hacer comparaciones y analizar los cambios seculares producidos en el dimorfismo sexual y la menarquía ${ }^{42}$.

\section{La evolución de la estatura de los varones en Francia y en España. Una perspectiva comparada}

En esta sección se compara la estatura de los hombres españoles con la de los franceses. Los datos de reclutamiento en ambos países permiten su reconstrucción al menos desde las generaciones nacidas en la década de 1830. Para el caso de Francia, hay datos de estaturas para el Antiguo Régimen $^{43}$, pero las principales investigaciones se han centrado a partir de las décadas centrales del siglo XIX. Han sido fundamentales los trabajos de la dilatada escuela de antropología física, primero, y de la renovada escuela de historia económica y social, después. De la primera destacan los estudios de Marie-Claude Chamla y sus colaboradores, y de los posteriores discípulos como Olivier ${ }^{44}$, Pineau y Demoulin ${ }^{45}$. De la segunda, hay que remitirse a los trabajos pioneros de Le Roy Ladurie y su equipo, a los que luego se sumaron los de los nuevos historiadores económicos, como Michel Van

\footnotetext{
42. Komlos; Baten, n. 2; Steckel; Floud, n. 34; Steckel, n. 2.

43. Komlos; Hau; Bourguinat, n. 36, p. 159 y ss. Heyberger, Laurent. Toward an anthropometric history of provincial France, 1780-1920. Economics and Human Biology. 2007, 5 (2): 229-254.

44. Olivier, Georges. Biologie humaine et régions françaises. Comptes-Rendus de l'Académie des Sciences de Paris. 1969; 268: 2085-2087. Olivier, Georges. The increase of stature in France. Journal of Human Evolution. 1980; 9: 645-649. Olivier, Georges; Chamla, Marie-Claude; Devigne, Georges; Jacquart, Albert; lagolnitzer, Edmond. L'accroissement de la stature en France II. Les causes du phénomène: analyse univariée. Bulletins et Mémoires de la Société d'Anthropologie de Paris. 1997; 4 (13): 205-214.

45. Pineau, Jean-Claude. La stature en France depuis un siècle: évolution générale et régionale. Bulletins et Mémoires de la Société d'Anthropologie de Paris. 1993; 5: 257-268. Demoulin, Françoise. Secular trend in France. In: Bodzsár, Éva B; Susanne, Charles, eds. Secular growth change in Europe. Budapest: Eötvös University Press; 1998, p. 109-134.
} 
Meerten y David Weir ${ }^{46}$, y más recientemente los de Gilles Postel-Vinay, David E. Sahn y Laurent Heyberger centrados sobre el siglo XIX y el primer tercio del siglo $\mathrm{XX}^{47}$. Algunos economistas han explorado las tallas de los franceses en los últimos tiempos ${ }^{48}$.

Para España, la serie de promedios de talla más larga y homogénea que disponemos es la relativa a las provincias que conforman el Levante español, compuesta por más de 400.000 observaciones de municipios de Almería a Castellón. Esta serie está compuesta con tallas de los mozos de reemplazos de 1857 a 1969. Analizada por cohortes, la serie comprende el periodo de 1837 a 1948. Se ha comprobado que el promedio de la estatura de la zona del Levante español puede extrapolarse al comportamiento de la talla de los varones españoles ${ }^{49}$. Por ello, la serie enlaza luego con la del conjunto de España, disponible desde los reemplazos de 1955 hasta 1999 (cohortes de 1934 a 1980) con datos estadísticos del Ministerio de Defensa y el INE ${ }^{50}$. De manera que con ambas series podemos tener una evolución bastante aproximada de la estatura masculina desde 1830 a 1980. Una aproximación a los periodos anteriores, para finales del siglo XVIII, la encontramos en las recientes estimaciones que se han realizado con las tallas de localidades rurales de la antigua provincia de Toledo y de

46. Van Meerten, Michel A. Développement économique et stature en France, XIXe-XXe siècles. Annales. Economies, Sociétés, Civilisations. 1990; 45: 755-778. Drukker, Jan W.; Van Meerten, Michel A. Beyond Villerme and Quetelet: the quantitative relation between sex-and-specific height height and real per capita income. In: Komlos, n. 3, p. 25-57. Weir, David R. Economic welfare and physical well-being in France, 1750-1990. In: Steckel, Richard. H; Floud, Roderick, eds. Health and welfare during industrialization. Chicago and London: The University of Chicago Press; 1997, p. 161-200.

47. Postel-Vinay, Gilles; Sahn, David E. Explaining stunting in nineteenth century France. Economic History Review. 2010; 63 (2): 315-334. Heyberger, Laurent. Santé et développement économique en France au XIX siècle. Essai d’histoire anthropométrique. Paris: L'Harmattan; 2003. Heyberger, n. 7. Ver el número monográfico «L'Histoire anthropométrique en France», publicado en la revista Histoire, Economie et Societé. 2009, 28 (1), coordinado por Laurent Heyberger.

48. Herpin, Nicolas. La taille des hommes: son incidence sur la vie en couple et la carrière professionnelle. Économie et Statistique. 2003; 361: 71-90.

49. Martínez Carrión, José Miguel. Calidad de vida y bienestar biológico en el largo plazo. El caso de España. In: Dobado, Rafael; Gómez Galvarriato, Aurora; Márquez, Graciela, comps. México y España: ¿Historias económicas paralelas? México: Fondo de Cultura Económica; 2007, p. 673-704. Puche-Gil, Javier. Evolución de los «niveles de vida biológicos» en la Comunidad Valenciana, 1840-1948. Sevilla: Universidad Pablo de Olavide de Sevilla; 2009.

50. Series del INE, ver: Quiroga, Gloria. Medidas antropométricas y condiciones de vida en la España del siglo XX. Universidad Alcalá de Henares; 2003. 
Andalucía oriental. Con alistamientos de principios del siglo XIX, como el realizado en 1808 con motivo de la Guerra de la Independencia, disponemos de información sobre la estatura durante las décadas de 1770-80. Ello nos permite situarnos a finales del Antiguo Régimen y explorar su impacto en el bienestar ${ }^{51}$. Debemos señalar que las tallas que aparecen en los alistamientos del antiguo régimen vienen registradas en pies, pulgadas y líneas. A diferencia de otros países, la conversión del pie español debe realizarse a partir del Pie de París, que es en el que está basado, y no en el Pie de Burgos, que era la medida castellana más común en la época. Este sistema de medición se impuso también en el imperio español a través de las instituciones de la monarquía hispana y permaneció vigente hasta la década de 1850. Desde 1856, la legislación reguló la medición de la talla de los mozos llamados a filas con el nuevo el sistema métrico, impuesto a escala internacional. Desde entonces y salvo algunas anomalías que prolongaron el sistema antiguo hasta la década de 1860, la estatura de los españoles se registró en centímetros y sobre todo en mílímetros ${ }^{52}$.

La serie de la estatura media masculina en Francia se ha realizado a partir de las estimaciones de David R. Weir, con datos del reclutamiento militar de 1804-1922, relativos a los nacidos de 1783-1902. Continúa con las estimaciones de Michael A. Van Meerten para las cohortes de 1903 a 1966, que el autor extrapola de la renta per capita, considerando que la estatura es un proxy de esta última. Aunque esta serie ha recibido críticas contundentes, comprobamos que las estimaciones no se alejan de las observadas en los estudios clásicos de Chamla, siendo aceptables al menos hasta 1940 (nacidos en 1920). Para la segunda mitad del siglo XX utilizamos las estimaciones de Olivier $(1985,1991)$ y de Pineau (1993), recogidas la mayor parte de ellas por Demoulin (1998), y las de Herpin (2003) para el tramo final. El periodo más controvertido es el que atañe a los reemplazos de la Segunda Guerra Mundial y de la posguerra francesa. Así, para ambos

51. Para las poblaciones de la provincia de Toledo, ver García-Montero, Héctor. Los niveles de vida en la España del antiguo régimen. Estado de la cuestión y propuestas de investigación. In: Chastagnaret, Gérard; Daumas, Jean Claude; Escudero, Antonio; Raveux, Olivier, eds. Los niveles de vida en España y Francia (siglos XVIII-XX). Alicante; Universidad de Alicante: 2010, p. 21-44. Para Andalucía oriental, ver Cámara-Hueso, Antonio David. Long-term trends in height in rural Eastern Andalucía (1750-1950). Historia Agraria. 2009; 47: 45-67.

52. Sobre la conversión de pies y pulgadas a centímetros, Cámara-Hueso, Antonio David. Fuentes antropométricas en España: problemas metodológicos para los siglos XVIII y XIX. Historia Agraria. 2006; 38: 105-118. 
países disponemos de series anuales que remontándose a finales del Antiguo régimen nos permiten hacer comparaciones.

Figura 1

Evolución de la estatura media de varones adultos, de 20-21 años, en España y Francia.

Cohortes de 1770 a 1980

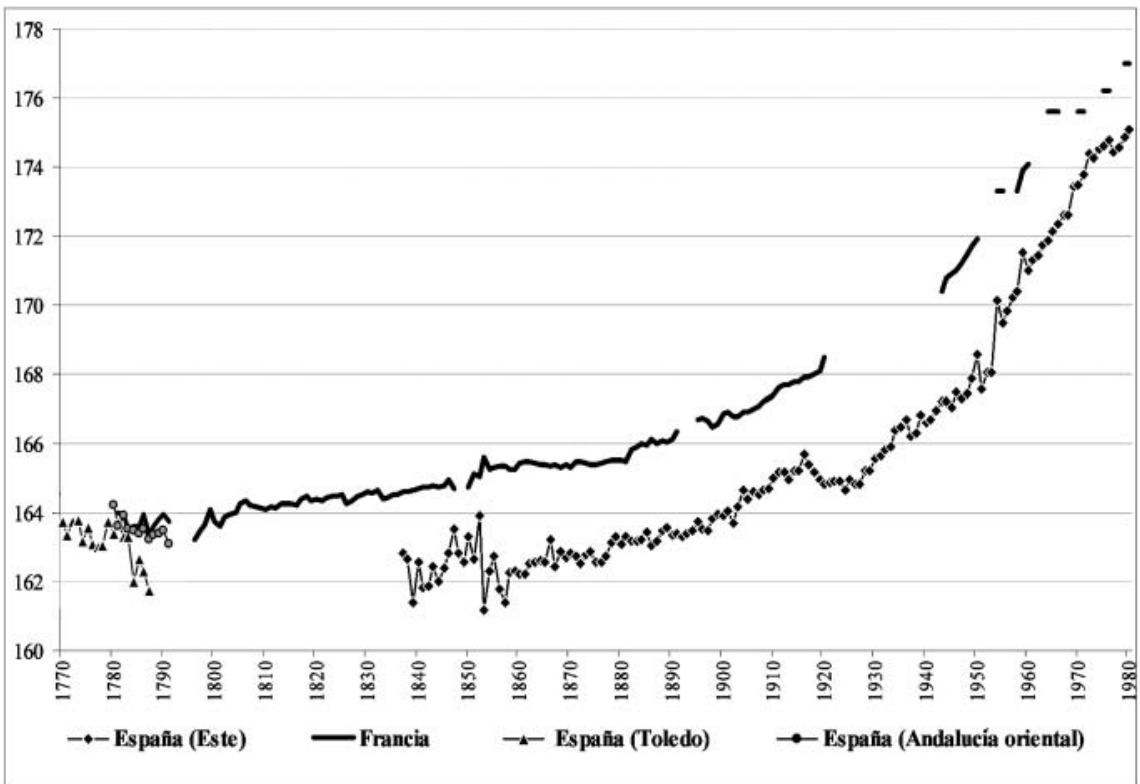

Fuente: Para Francia: Weir, 1997, n. 46; Van Meerten, 1990, n. 46; Olivier, 1980, n. 44; Pineau, 1993, n. 45; Demoulin, 1998, n. 45 y Herpin, 2003, n. 48. Para España, Cámara-Hueso, 2009, n. 51; García-Montero, 2010, n. 51; Quiroga, 2002, n. 50 y Actas de reclutamiento de archivos locales, reemplazos de 1857 a 1969 (nacidos de 1837 a 1948 de 21 municipios del Levante mediterráneo). Elaboración propia.

Como se muestra en la Figura 1, la estatura media de los varones jóvenes españoles no se diferenciaba mucho de la alcanzada por los franceses a finales del siglo XVIII. Los promedios estaban en torno a 163-164 cm, similares en 1780 y no muy diferentes de los promedios de otros países europeos por esos años. No obstante, las estimaciones españolas para las décadas de 177080 deben tomarse con cuatela pues son datos principalmente del mundo rural, de localidades del interior castellano y de la montaña de Andalucía oriental, por lo que se requiere más investigación. Llama la atención que en las dos series disponibles para esos años se advierte una tendencia a la 
caída de la estatura media en consonancia con los problemas demográficos y económicos que se han señalado para finales del siglo XVIII ${ }^{53}$.

Hasta las cohortes de finales de la década de 1830 no tenemos información antropométrica. Pero las estimaciones que presentamos (Figura 1) y otras de diferentes áreas muestran que las diferencias con Francia no fueron significativas, cifrándose en apenas un centímetro o dos en la década siguiente. Los datos sugieren que frente a las pequeñas ganancias observadas en Francia en la primera mitad del siglo XIX, algo más de un centímetro, en España hubo un ligero deterioro - estimado también en más de un centímetro- como consecuencia probablemente de los efectos que la prolongada crisis del Antiguo Régimen tuvo en las economías familiares campesinas $^{54}$. El hecho requiere más investigación antropométrica. Hacia las décadas de 1850-60, ambos países se hallan en un periodo de transición de las economías agrarias tradicionales a las economías modernas, aunque en Francia el proceso de industrialización se encuentra más avanzado. En contraste con España, la industria gala prospera de forma sostenida a partir de esas fechas y también lo hace la renta per capita de la población francesa ${ }^{55}$. La evolución de la estatura expresa un estado nutricional neto favorable para Francia desde las cohortes de 1850. Los reclutas franceses muestran tallas medias superiores a las de los españoles en torno a tres centímetros, valores que se estabilizaron en el curso de la segunda mitad del siglo XIX y se mantuvieron hasta el final del siglo XX, tramo en el que los españoles crecieron más deprisa y acortaron distancias con respecto a los franceses.

Al final del periodo analizado (cohortes de 1980), la talla promedio de los españoles está por encima de $\operatorname{los} 175 \mathrm{~cm}$ y la de los franceses

53. Domínguez Martín, Rafael. Niveles de vida e indicadores de bienestar social a finales del Antiguo Régimen: comparaciones internacionales y contrastes regionales. In: Llopis Agelán, Enrique, ed. El legado económico del Antiguo Régimen en España, Barcelona, Crítica; 2004, p. 321-327. Moreno Lázaro, Javier. ¿Fomentó el capitalismo agrario la desigualdad? Salarios y niveles de vida en Castilla la Vieja, 1751-1861. In: Martínez Carrión, José Miguel, ed. El nivel de vida en la España rural, siglos XVIII-XX, Alicante, Publicaciones de la Universidad de Alicante; 2002, p. 75-112.

54. Sobre el nivel de vida de las poblaciones campesinas, ver Martínez Carrión, José Miguel, ed. El nivel de vida en la España rural, siglos XVIII-XX, Alicante, Publicaciones de la Universidad de Alicante; 2002.

55. Martínez Carrión, José Miguel. Sobre el atraso económico de España. In: Barciela, Carlos; Chastagneret, Gérad; Escudero, Escudero, eds. La historia económica en España y Francia. Siglos XIX y XX. Alicante: Publicaciones de la Universidad de Alicante; 2006, p. 351-403. 
alcanza $177 \mathrm{~cm}$. Si tomamos como punto de partida el decenio de 1780 (talla promedio cohortes en España $=163,3 \mathrm{~cm}$ y Francia $=163,7 \mathrm{~cm}$ ), las diferencias son practicamente inexistentes a finalizar el siglo de las Luces, mientras que son algo significativas al finalizar el siglo XX, al cabo de dos siglos. El incremento, siendo notable en ambos casos, ha sido mayor en Francia que en España. Mientras los varones franceses aumentan su talla promedio algo más de $13 \mathrm{~cm}$, los españoles aumentan $12 \mathrm{~cm}$. La mayor diferencia entre la talla de ambos países se produce en la primera mitad del siglo XIX. El modo en que ambos países salieron de la crisis del Antiguo Régimen y transitaron al liberalismo pudo estar en el origen del gap que observamos a mediados del siglo XIX en la Figura 2. Los resultados sugieren que los cambios institucionales que regularon los derechos de propiedad y de acceso a los recursos básicos, como la educación y la sanidad, fueron fundamentales. En el caso de Francia, la quiebra de la monarquía absoluta y el cambio institucional se produjo de forma «revolucionaria» con el cambio de siglo, y desde luego no se prolongó tanto en el tiempo, como ocurrió en España, que perduró hasta 1833, con elevados costes económicos y sociales como ha mostrado la historiografía. Con la evidencia antropométrica que presentamos, además sabemos que la transición al nuevo régimen liberal tuvo un alto coste en términos de bienestar biológico.

De la Figura 1 se infiere que la tendencia de la estatura no fue unidireccional en el curso del tiempo, sino que estuvo sometida a fluctuaciones y deterioros. En el caso de España se detectan varias etapas: a) deterioro desde finales del siglo XVIII hasta las décadas centrales del siglo XIX, aunque esta etapa está insuficientemente analizada; b) estancamiento entre los nacidos durante el reinado de Isabel II y el Sexenio Revolucionario, principalmente entre 1850 y 1875, con episodios de deterioro entre 1845 y 1855 ; c) recuperación y crecimiento a partir de la segunda mitad de la década de 1870, con avances durante la Restauración que se prolongaron hasta las cohortes nacidas en la Primera Guerra Mundial; d) deterioro entre 1917 y 1930 y que corresponde a los reemplazos que vivieron la Guerra Civil y la posguerra en su fase del estirón adolescente: la talla media disminuyó un centímetro; e) finalmente, fuerte crecimiento que arrancó con los nacidos en los primeros años de la década de 1930 y se prolongó hasta las décadas de 1960 y 1970, un periodo sustancial de progreso del nivel de vida biológico y del bienestar en general de los españoles. 
Figura 2

Diferencias de estatura en cms. de varones adultos en España y Francia. Cohortes de 1835 a 1980

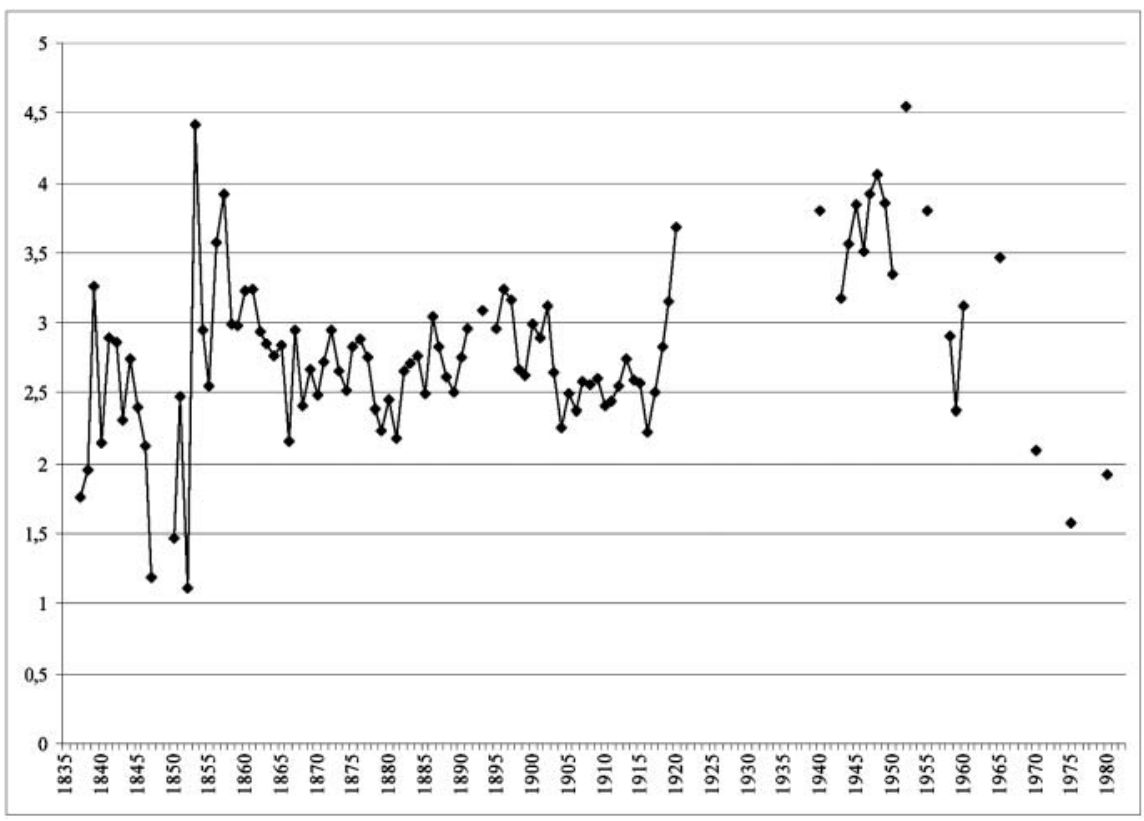

Fuente: Para Francia: Weir, 1997, n. 46; Van Meerten, 1990, n. 46; Olivier, 1980, n. 44; Pineau, 1993, n. 45; Demoulin, 1998, n. 45; y Herpin, 2003, n. 48. Para España; Quiroga, 2002, n. 50. Elaboración propia.

En contraste con las tallas de España, Francia muestra un ritmo de crecimiento sostenido de la estatura durante el siglo XIX, consecuencia de las mejoras relativas del bienestar asociado al bajo crecimiento demográfico - presenta las tasas de natalidad más bajas de Europa y muestra, también, un descenso de la mortalidad cuya tasa era también relativamente más baja que la de España-, unas condiciones de tamaño óptimo de las explotaciones campesinas y el lento afianzamiento de la industrialización. La urbanización no supuso graves problemas al bienestar biológico como se ha visto en los países europeos más industrializados, caso de Inglaterra. Y tampoco registra emigración como en otras partes de Europa. En el siglo XX, la estatura y otros indicadores del nivel de vida muestran progresos acelerados ${ }^{56}$. España 
se distancia de Francia desde 1850 y presenta una diferencia de casi tres centímetros que se mantiene casi estable hasta la década de 1960, a partir de la cual se acortan distancias (Figura 2).

El episodio más negro de la tendencia secular para ambos países se observa en los reclutamientos de las guerras: la Guerra Civil (1936-39) en el caso de España y de la Segunda Guerra Mundial (1939-45) en el caso de Francia. Sin embargo, para el caso de Francia la estimación de la talla deriva de una extrapolación de la renta, lo cual hace que la estatura caiga significativamente en los años del conflicto mundial, un hecho que resulta poco probable, por lo que hemos evitado incorporar los resultados a la Figura 1. En cambio, en España los datos muestran un descenso de la altura de los reemplazos de 1937 a $1947^{57}$. El impacto de la Guerra Civil y sobre todo de la posguerra, durante la autarquía franquista, se estima en la caída de más de un centímetro de talla entre las cohortes de 1916 y 1930, que fueron las que vivieron situaciones de penuria coincidiendo con su estirón adolescente. El hambre y la malnutrición en la década de 1940, al menos hasta 1947, segó el crecimiento potencial que pudo haberse desarrollado de haber existido normalidad en el suministro y consumo alimentario. La situación de privación relativa y la pobreza persistieron en España hasta finales de los década de 1940, etapa en la que además se recrudecieron las enfermedades y las desigualdades ante la muerte ${ }^{58}$. Los efectos también debieron de notarse entre los nacidos en esos años, pero las mejoras del bienestar producidas en las décadas siguientes posibilitaron la recuperación del crecimiento al final de la adolescencia.

La estatura que alcanzaron las cohortes de la década de 1930 se recupera hacia 1950. Los datos sugieren que las mejoras del bienestar producidas en España son significativas, sobre todo a partir de los años cincuenta en que los indicadores del nivel de vida alcanzaron tasas de crecimento elevadas:

57. Sobre el impacto de la autarquía en las tallas de la población de la Comunidad Valenciana, ver Puche-Gil, Javier. Guerra Civil, autarquía franquista y bienestar biológico en el mundo rural valenciano (1936-1949). Historia Agraria. 2010; 52: 121-147.

58. Del Cura, María Isabel; Huertas, Rafael. Alimentación y enfermedad en tiempos de hambre: España, 1937-1947. Madrid: Consejo Superior de Investigaciones Científicas; 2007. Bernabeu Mestre, Josep; Caballero Pérez, Pablo; Galiana Sánchez, María Eugenia; Nolasco, Andreu. Niveles de vida y salud en la España del primer franquismo: Las desigualdades en la mortalidad infantil. Revista de Demografía Histórica. 2006; 24 (1): 181-202. 
renta per cápita, consumo ${ }^{59}$, educación, esperanza de vida, o relativamente bajas como mortalidad infantil ${ }^{60}$. Los avances de la década de 1950 y el fuerte crecimiento económico de 1960 a 1975 favorecieron un ambiente favorable para el incremento de la estatura que de forma notoria se observa en los reclutamientos de los años 50 en adelante. El aumento del nivel de vida biológico es general a partir de las cohortes de la Segunda Guerra Mundial, sobre todo en las décadas de 1960-1970, y se advierte un tímido proceso de convergencia con los promedios de talla de Francia y del resto del mundo desarrollado. Las cohortes de 1948 mostraron una diferencia de talla de más de $4 \mathrm{~cm}$ a favor de Francia, que aumentó a 4,5 cm en 1952, para disminuir posteriormente; en 1975, la diferencia era sólo de 1,5 cm. Para las generaciones de 1980, la talla media masculina alcanzó los 177 cm en Francia y superó los $175 \mathrm{~cm}$ en España.

La convergencia de los promedios de la estatura de los españoles con la de los franceses y el resto de los europeos tiende a producirse en las últimas décadas del siglo XX, aunque no tan intenso como el observado entre las tallas medias de las regiones españolas ${ }^{61}$. Entre 1930 y 1970, las cohortes españoles crecieron algo más de 8 centímetros, con un crecimiento medio anual muy superior al registrado en la mayor parte de los países europeos. Este fuerte incremento se debió al tirón que ejerció el crecimiento económico en el bienestar desde 1960, pero también a la «recuperación» del potencial físico logrado en tiempos de la preguerra.

El aumento del nivel de vida, el incremento de la renta por habitante y del consumo alimenticio junto a las mejoras de la salud son factores esenciales del crecimiento humano en el siglo $\mathrm{XX}^{62}$. Las recientes investi-

59. El incremento del consumo de leche se produce desde finales del siglo XIX, Nicolau-Nos, Roser; Pujol-Andreu, Josep; Hernández, Ismael. Milk, social acceptance of a new food in Europe: Catalonia, 19th-20th centuries. Dynamis. 2010; 30: 119-141. Sobre la transición nutricional y los cambios del consumo alimenticio en España, ver Cussó, Xavier. El estado nutritivo de la población española 1900-1970. Análisis de las necesidades y las disponibilidades de nutrientes. Historia Agraria. 2005; 36: 329-358. Cussó, Xavier; Garrabou, Ramón. La transición nutricional en la España contemporánea: las variaciones en el consumo de pan, patatas y legumbres (1850-2000). Investigaciones de Historia Económica. 2007; 7: 69-100.

60. Sobre la evolución de la economía española, Carreras, Albert; Tafunell, Xavier. Historia económica de la España contemporánea. Barcelona: Crítica; 2010. Prados de la Escosura, Leandro. El progreso económico de España (1850-2000). Madrid: Fundación BBVA; 2003.

61. Martínez-Carrión, n. 49.

62. Sobre las mejoras de la salud, ver artículos publicados en esta revista. A guisa de ejemplo: Huertas García-Alejo, Rafael. Salud Pública en la España contemporánea. Dynamis. 1994; 14: 
gaciones sugieren que las mejoras nutricionales han sido importantes en el siglo pasado y advierten una relación entre el incremento del consumo de proteínas animales, como la carne, la leche y los huevos, entre otros componentes nutricionales, y el crecimiento de la estatura ${ }^{63}$.

\section{Conclusiones}

Durante la primera mitad del siglo XIX la antropología física francesa experimentó un avance similar al que tuvo lugar en España a finales de la centuria. Hasta comienzos del siglo XX los estudios realizados sobre la talla y sus determinantes privilegiaron los factores genéticos y de raza. La situación comenzó a cambiar significativamente en el curso de la primera mitad del siglo XX, y desde la década de 1960 se fueron imponiendo las tesis que otorgaban importancia a los factores ambientales y socioeconómicos. La antropología francesa tuvo un papel protagonista con la escuela de Chamla, que tuvo repercusiones en España. En las últimas décadas del siglo XX la historia antropométrica se consolidó por los trabajos de los historiadores económicos, principalmente, que han aportado evidencia sobre la importancia que tienen los determinantes sociales y económicos en la evolución de la estatura.

La evolución de la talla humana en el largo plazo dependió más de factores ambientales que de factores genéticos. Los resultados que presentamos afirman la importancia que tiene el estudio del crecimiento humano para el debate de los niveles de vida y el bienestar en general. Aunque la genética influye al ser modificada por los movimientos migratorios que fueron intensos en el curso del siglo XX, los cambios ambientales explican buena parte del crecimiento humano en ambos países. El planteamiento sugiere la importancia que pueden tener las políticas públicas de salud en la mejora del estado nutricional.

17-21; y los estudios de los investigadores Josep Lluis Barona, Josep Bernabeu-Mestre, Esteban Rodríguez Ocaña y Enrique Perdiguero, entre otros. Recientemente, en Nicolau, Roser. La mortalidad en España en los siglos XIX y XX. Una comparación con Francia. In: Chastagnaret, Gérard; Daumas, Jean Claude; Escudero, Antonio; Raveux, Olivier, eds. Los niveles de vida en España y Francia (Siglos XVIII-XX). Alicante: Universidad de Alicante; 2010, p. 129-146.

63. Para los países de la Europa occidental: Susanne, Charles; Bodzsar, Éva; Bielicki, Tadeusz et al. Changements séculaires de la croissance et du développement en Europe. Antropo. 2005; 0: 71-90. Para Francia, ver Demoulin, n. 45, p. 109-134. Para España, ver Rebato, n. 27. 
En este artículo se demuestra que los españoles han sido más bajos que los franceses en el curso de los dos últimos siglos, aunque partieron de promedios de estatura muy cercanos a finales del siglo XVIII y se vuelven a aproximar en las últimas décadas del siglo XX. La talla de los españoles creció más de 11 centímetros entre las cohortes de 1855/60 y 1970, mientras que la talla media de los franceses creció 9,5 cm en el mismo periodo. Al final del siglo XX la talla de los españoles tiende a converger más que otras poblaciones europeas en un proceso equivalente al observado en la renta per cápita. Pese a los avances, queda margen para el progreso del bienestar biológico.

Nuestras pesquisas muestran la influencia de los conflictos bélicos en el bienestar humano. En el curso del siglo XX España y Francia, respectivamente, sufrieron las terribles consecuencias de la Guerra Civil de 1936-1939 y de la Segunda Guerra Mundial. Los datos sugieren que los conflictos bélicos y las políticas autárquicas fueron los peores aliados del crecimiento y del bienestar humano al deteriorarse el estado nutricional. El hecho no está suficientemente probado en Francia, pese a la extrapolación que plantea Van Meerten; en cambio, está bien documentado en España. La talla promedio disminuyó por las secuelas que ocasionó la Guerra Civil y sobre todo por las políticas económicas de la autarquía en la inmediata posguerra. Estas sumieron a la población española en el aislamiento y la penuria económica. Por ahora, los ciclos de mayor deterioro se observan en las cohortes nacidas entre 1915 y 1930, que fueron las que vivieron el conflicto bélico y la posguerra.

Junto a la tendencia secular de claro signo ascendente en el curso del siglo XX, la existencia de ciclos en el bienestar biológico ha constituido una de las principales aportaciones de la historia antropométrica. El caso español es un claro ejemplo. En general, el aumento de la estatura observado desde finales del siglo XIX y el fuerte crecimiento registrado tras la Segunda Guerra Mundial revelan la intensidad del progreso económico y la mejora sustancial de los estándares biológicos. En ambos países, el avance del bienestar humano coincide con los procesos de modernización económica y el desarrollo de las transiciones demográfica, epidemiológica y nutricional. Las primeras tienen sus mayores logros en el declive de la mortalidad infantil y el incremento de la esperanza de vida, como consecuencia de los avances tecnológicos y la intervención de las instituciones en la mejora de la salud pública. La última transición posibilitó el paso de unas dietas empobrecidas e insuficientes para cubrir los requerimientos 
nutricionales a otras mucho más equilibradas y de mayor calidad, pese al incremento de la obesidad en los últimos tiempos. Desde 1970, el incremento de las estaturas corre parejo al proceso de convergencia instalado en buena parte de las regiones del sur europeo y ha hecho posible que las tallas medias de las regiones pobres alcancen los estándares del mundo desarrollado. A comienzos del siglo XXI la talla media de los varones adultos españoles, estimada en torno a 176 centímetros, se encuentra un centímetro por encima del promedio europeo, supera a la de los italianos y se acerca a la talla media de los franceses. 